\title{
PFG-NMR ON DOUBLE EMULSIONS: A DETAILED LOOK INTO MOLECULAR PROCESSES
}

\author{
R. Bernewitz ${ }^{1}$, X. Guan ${ }^{1}$, G. Guthausen ${ }^{1}$, F. Wolf ${ }^{2}$ and H. P. Schuchmann ${ }^{2}$ \\ KIT, D-76131 Karlsruhe, Germany \\ ${ }^{1}$ SRG10-2, Institute for Mechanical Engineering and Mechanics \\ ${ }^{2}$ Institute of Process Engineering in Life Sciences, Section I: Food Process Engineering
}

\section{INTRODUCTION}

Single emulsions are of high interest in different fields and therefore well investigated. They provide more advantages when an emulsion is part of another emulsion, as double- or multiple emulsion. Multiple emulsions, as novel food systems, reduce the amount of fat in food or encapsulate active substances. They are not only of interest for food, but also for pharmaceutical or cosmetic products.

As in case of single emulsions, multi-modality and droplet size distribution (DSD) of multiple emulsions are important parameters for product quality and physical properties (e.g. viscosity and shelf life). These parameters are also important to gain insight into mechanisms taking place in the second step of the two-step production of double emulsions, which still is not understood in detail. Currently, common measurement techniques for the determination of DSD and its modality of single emulsions are Laser light scattering, Laser diffraction, Ultra sonic attenuation and PFG-NMR. However, acoustic and optical methods do not work properly or not even at all, when aiming for information about structure properties of the inner emulsions. Additionally, results on the outer emulsion are not reliable anymore when using the optical methods, due to multiple diffraction inside the double emulsion.

PFG-NMR is a well established method to determine the droplet size distribution of single emulsions. It is known to work non-destructive with a small workload and a high selectivity. In this context, high selectivity means, that the optical properties of the media do not play a role. Using ${ }^{1} \mathrm{H}-\mathrm{NMR}$ for example, only molecules containing hydrogen can be detected. These molecules can be differentiated by their specific chemical shift in high resolution NMR, or by relaxation filters as applied in low filed NMR.

Therefore, the PFG-NMR method is in principle suitable to determine structural parameters like DSD in double emulsions. However, the current restrictions have to be exploited, and new models have to be created to analyze the signal decays measured on double emulsions. Molecular processes like diffusion phenomena, relaxation and spectral properties have to be understood in order to obtain a correct interpretation of the data and quantitative meaningful double emulsion's DSD. 


\section{METHOD AND RESULTS}

\subsection{Methods}

2.1.1 Preparation of $W_{1} / O / W_{2}$ Double Emulsions. The double emulsions were produced in a two-step process: First, the inner emulsion was produced, using a tooth rim dispersing device. The inner emulsion consisted of water $\mathrm{W}_{1}$ with $0.5 \%$ gelatine and vegetable oil with 10\% PGPR90. In the second step, the inner emulsion was further emulsified by a colloid mill with water $\mathrm{W}_{2}$ containing 2\% LEO-10 and, recipe depended, $0.6 \%$ Xanthan. The typical range of mean oil droplets size is $d_{50,3}=14 \mu \mathrm{m}$, and of $\mathrm{W}_{1}$ droplets $d_{50,3}=6 \mu \mathrm{m}$.

In this work, double emulsions of the type $\mathrm{W}_{1} / \mathrm{O} / \mathrm{W}_{2}$ with a disperse phase ratio of $15 \% / 35 \% / 50 \%$ (Figure 1a)) were investigated by PFG-STE NMR experiments (Figure 1b)) and confocal laser scanning microscopy (CLSM).

2.1.2 PFG-STE NMR on $W_{1} / O / W_{2}$ Double Emulsions. The NMR experiments were performed on a Bruker Avance 200 SWB. The parameters of the PFG-STE sequence are summarized in Table 1. The sequence is known to be a suitable method to measure molecular diffusion phenomena. In comparison to a PFG-SE Hahn-Echo experiment, it allows longer diffusion times $\Delta$, due to the " $T_{2}$-bypass" after the second gradient pulse so that the diffusion time is restricted by $T_{1}$. PFG-NMR measures the displacement of molecules during $\Delta$. In pure substances, the diffusion of molecules is described by random movement, with a final displacement $\Delta r(\Delta),{ }^{1-3}$. In confining geometries, like in an emulsion's droplets, the mean free path differs from the one observed at free self-diffusion. Therefore, molecules exposed to restricted diffusion exhibit a different PFG-NMR signal. Summarizing, molecules in confined geometries show a different diffusion behaviour than molecules in the pure substance.

NMR data of single emulsions are most commonly evaluated with the model of Murday and Cotts / von Neuman (MC) ${ }^{3,4}$. This model is valid for single emulsions of water and oil, containing spherical droplets with a mean diameter $d_{50,3}>1 \mu \mathrm{m}$, assuming the Gaussian phase approximation. The question is, if data of double emulsions are modelled correctly by this model.
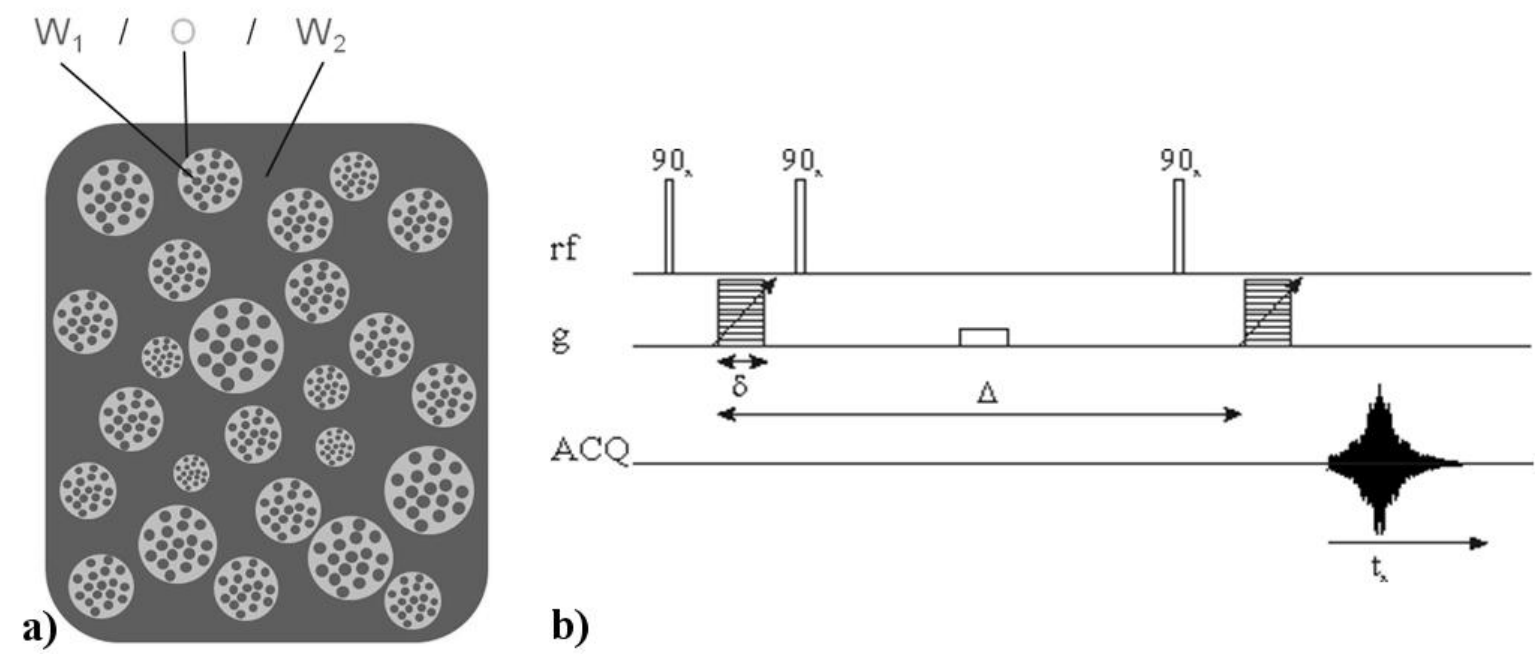

b)

Figure 1 a) Scheme of a $W_{1} / O / W_{2}$ double emulsion b) PFG-STE NMR sequence. 
Table 1 Parameters and their values of NMR experiments on a Bruker Avance 200

\begin{tabular}{lcl}
\hline Symbol & Parameter & Typical Value(s) \\
\hline$\delta$ & Gradient pulse duration & $4 \mathrm{~ms}$ \\
$\Delta$ & Diffusion time & $50,100,150,200,300,500 \mathrm{~ms}$ \\
$P_{90}$ & $90^{\circ}$ rf-pulse length & $10 \mu \mathrm{s}$ at $0 \mathrm{~dB}$ \\
$\tau_{1}$ & First rf-pulse delay & $6.115 \mathrm{~ms}$ \\
$D_{1}$ & Repetition time & $8 \mathrm{~s}$ \\
$g$ & Amplitude of magnetic gradient & $3.54 \mathrm{mT} / \mathrm{m} \ldots 5.99 \mathrm{~T} / \mathrm{m} \mathrm{linear} \mathrm{in} \mathrm{32}$ \\
& & steps; 3.54 mT/m... 22 $\mathrm{mT} / \mathrm{m}\left(\mathrm{W}_{2}\right)$ \\
$G$ & Amplitude of spoiler gradient & $0.2 \mathrm{~T} / \mathrm{m}$ \\
& Duration of spoiler gradient & $5 \mathrm{~ms}$ \\
& Rise time for gradient & $100 \mu \mathrm{s}$ \\
& Time for gradient stabilisation & $2 \mathrm{~ms}$ \\
$\mathrm{NS}$ & Number of scans & 2 \\
$\mathrm{DS}$ & Number of dummy scans & 2 \\
$\omega_{0}=2 \pi v_{0}$ & Larmor-Frequency & $2 \pi * 200.145 \mathrm{MHz}$ \\
& Spectral width & $20161 \mathrm{~Hz}$ \\
& Number of complex data points & 4096 \\
\hline
\end{tabular}

2.1.3 CLSM on $\mathrm{W}_{1} / \mathrm{O} / \mathrm{W}_{2}$ Double Emulsions. The double emulsions were additionally investigated by a CLSM in order to compare NMR results to a more direct method. To obtain a contrast between the three phases the oil phase was dyed with Rhodamine-B. CLSM was used because of its $2.5 \mathrm{D}$-capability. This allows a scan of the sample slice by slice.

\subsection{Results}

2.2.1 NMR Signal Attenuation of Double Emulsions. The spectra of a double emulsion, measured with $\Delta=0.3 \mathrm{~s}$, exhibit specific resonances of water (at $4.6 \mathrm{ppm}$ ) and oil (Figure 2a)). The investigated double emulsion $\mathrm{W}_{1} / \mathrm{O} / \mathrm{W}_{2}$ was prepared as described in 2.1.1 with the speciality that $\mathrm{W}_{2}$ didn't contain Xanthan. The spectrum amplitudes depend on the gradient amplitude as it is expected for signal from diffusing molecules. In Figure 2b) the logarithmic signal attenuation of the double emulsion is shown together with the logarithmic signal attenuations of $\mathrm{W}_{1}$ and $\mathrm{W}_{1} / \mathrm{O}$ single emulsion, which were retained during the production process.

The pure water phase $\mathrm{W}_{1}$ only shows a signal near $g=0 \mathrm{~T} / \mathrm{m}$ (Figure $2 \mathrm{~b}$ ), $\bullet$ ). This is due to free self-diffusion of water molecules in $\mathrm{W}_{1}$. The diffusion coefficient is in accordance with the water self diffusion at the measurement temperature of $22^{\circ} \mathrm{C}$.

The NMR data of the single emulsion, shown in figure $2 \mathrm{~b}$ ), was modelled with the MCmodel. Assuming a log-normal distribution function for the DSD, it revealed a median $d_{50,3} \approx 3.5 \mu \mathrm{m}$ with a width of $\sigma \approx 0.5$ of the corresponding Gaussian distribution. By a Coulter Counter LS230 laser diffraction device, the median of the single emulsion's DSD was determined to $d_{50,3} \approx 2 \mu \mathrm{m}$. Comparing both results, the difference of $d_{50,3}$ is within the mean standard deviation specified on the Coulter Counter. 
a)

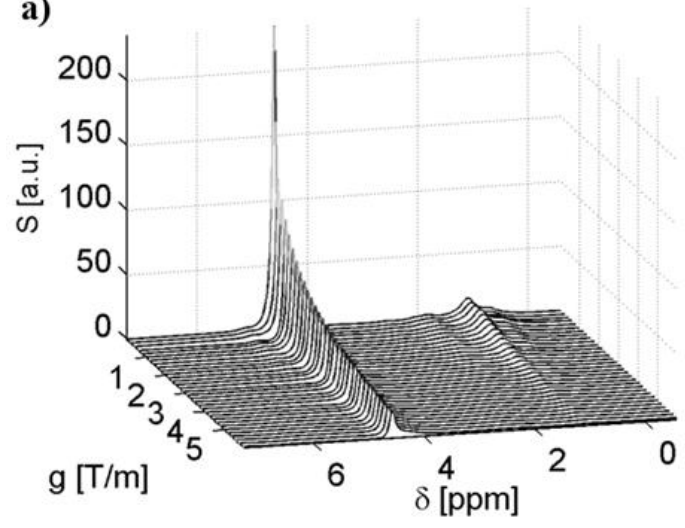

b)

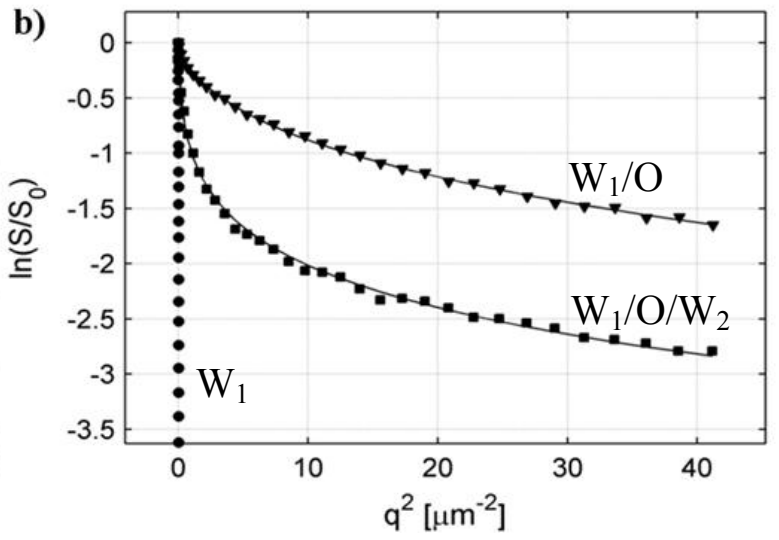

Figure 2 a) Spectrum of a $W_{1} / O / W_{2}$ double emulsion as a function of $g$.

b) Logarithmic signal attenuation of pure inner water phase $W_{1}$, the $W_{1} / O$ single emulsion after first emulsion step and the double emulsion.

However, the signal attenuation of the double emulsion differs significantly from the attenuation of the single emulsion. Modelling of the data with the MC-model leads to $d_{50,3} \approx 7 \mu \mathrm{m}$ with a width of $\sigma \approx 0.75$. Assuming a complete preservation of the simple emulsion's properties this DSD is physically meaningless. In the analysis, the whole water signal was considered and lead to the wrong DSD.

But there are possible reasons for the failure of the data modelling:

- Also fractions of $\mathrm{W}_{2}$ following the laws of restricted diffusion could have influenced the signal attenuation.

- Diffusion between the two water phases could occur, which leads to an imprecise separation of the signal of the two water phases. This means, diffusion takes place through the oil phase, which is called molecular exchange in our context. The bounds of the oil droplets function as a thin membrane in this picture. This phenomenon is also described for different inhomogeneous systems ${ }^{5-10}$.

2.2.2 Influence of restriction on water diffusion in $W_{2}$. In Figure 3 , the signal attenuations of $\mathrm{O} / \mathrm{W}$ single emulsions are shown as a function of the disperse phase concentration. The emulsions were measured via PFG-STE-NMR at $200 \mathrm{MHz}$ with $\Delta=0.05 \mathrm{~s}$. The disperse phase ratio was varied from $0 \%$ to $50 \%$. $0 \%$ means pure continuous phase, consisting of water with $2 \%$ Leo 10 and $0.2 \%$ Xanthan. 


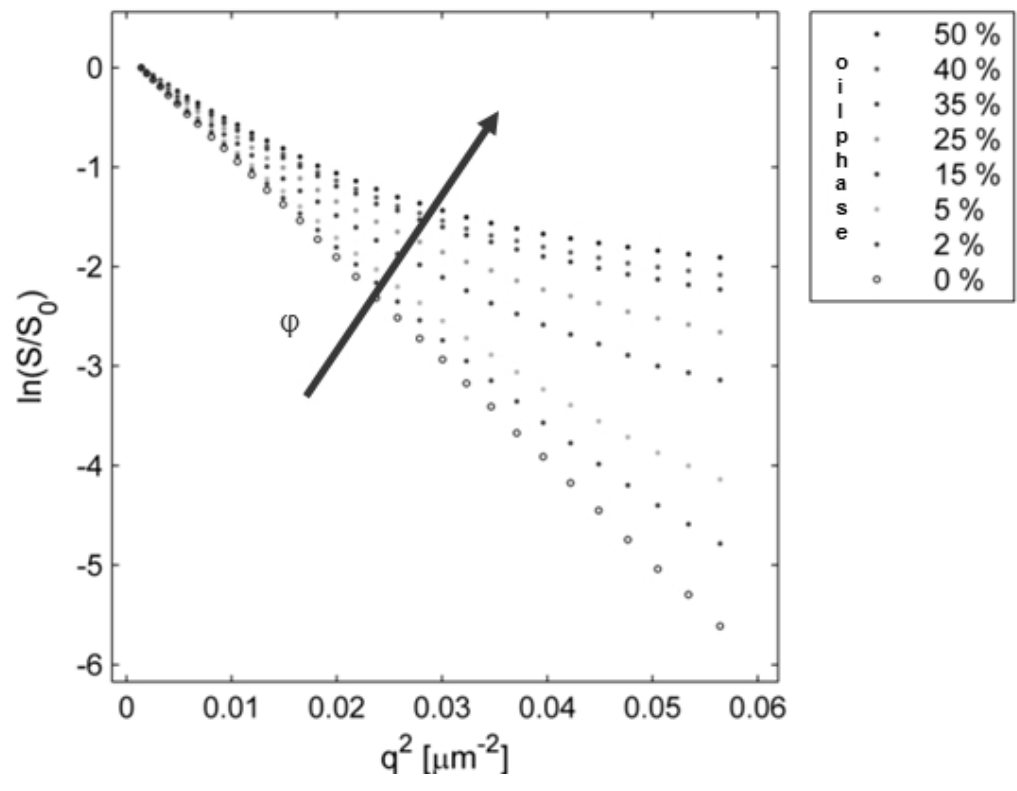

Figure 3 Signal attenuation of $O / W$ emulsions with different disperse phase ratios $\varphi$.

The logarithmic signal attenuation of the pure continuous phase is a linear function of $q^{2}$, which was expected because of free self-diffusion. The curvature of $\ln \left(S / S_{0}\right)$ increases with growing disperse phase ratio, because of increasing non-spherical restriction of the water molecules between the oil droplets. However, taking the small range of $q^{2}$ into account, the influence of this effect is marginal. Regarding the range of $q^{2}$ of common PFG-NMR experiments (Figure $2 b$ ), the effect can be neglected for $\mathrm{W}_{1}$ diffusional behaviour.

Concluding, the outer phases in single emulsions as well as the outermost phase in double emulsions have to be considered as following restricted diffusion even at very small disperse phase concentrations.

2.2.3. Diffusion Phenomena between $W_{1}$ and $W_{2}$ in $W_{1} / O / W_{2}$. In several publications, the possibility of molecular exchange in double emulsions is discussed ${ }^{5-12}$. In some publications $^{8-10}$, a molecular exchange model was applied, which originally was found to be applicable in case of cellular systems ${ }^{5-7}$. There is, however, some controversy whether such effects are observed or other phenomena are responsible for the experimental results. One argument against the exchange hypothesis is that the solubility of the two substances is low and they tend to separate when mixed.

To find out if and on which time scale diffusion between $\mathrm{W}_{1}$ and $\mathrm{W}_{2}$ takes place ${ }^{11}$, the idea was to add a small amount of Xanthan to $\mathrm{W}_{2}$. Xanthan is a common thickener, also used in food industry. It increases the viscosity and consequently decreases the mobility of molecules. In Figure 4a) NMR data obtained from a double emulsion with Xanthan in $\mathrm{W}_{2}$ is shown. In comparison to a double emulsion without Xanthan (Figure 2a)), a sudden drop occurs after the first gradient step. This difference could be explained by decreased exchange between $\mathrm{W}_{1}$ and $\mathrm{W}_{2}$. To verify this suspicion, another experiment was performed, using driven diffusion effects.

Right before the NMR experiment, the $\mathrm{W}_{1} / \mathrm{O} / \mathrm{W}_{2}$ double emulsions with Xanthan in $\mathrm{W}_{2}$ were diluted with additional $50 \% \mathrm{~W}_{2}$, containing different amounts of sugar $(1 \%, 5 \%$, 
$15 \%$ ). To avoid further large energy input, the additional phase was added by hand while stirring with a glass stick.

As shown in (Figure $4 \mathrm{~b}$ )-d)), the signals at $g>0 \mathrm{~T} / \mathrm{m}$ disappear with increasing sugar concentration. By adding sugar, the equilibrium of exchange was influenced, and driven diffusion from $\mathrm{W}_{1}$ to $\mathrm{W}_{2}$ took place. The process continues on a short time scale until a new equilibrium between the two phases is established. Regarding this fact, the conclusion is that, despite of Xanthan in the outer water phase, molecular exchange between $\mathrm{W}_{1}$ and $\mathrm{W}_{2}$ occurs. However, the exchange rate is that low that it is negligible on the time scale of the NMR experiments. This observation is proofed by pictures made with a CLSM, shown in Figure 5.

Here, a double emulsion with Xanthan in $\mathrm{W}_{2}$ was recorded before adding additional $\mathrm{W}_{2}$, containing $15 \%$ sugar. The process was observed online while adding the phase. During the disappearance of $\mathrm{W}_{1}$ it was observed in the CLSM experiments that the $\mathrm{W}_{1}$-droplets near the interface of the oil droplets to $\mathrm{W}_{2}$ tend to move fast along the boundary of the oil droplet. In contrast to that, the $\mathrm{W}_{1}$-droplets in the centre of the oil droplets seem not to be influenced by the sugar in the outer water phase and do not move on a short time scale.

The impression is that the exchange processes start from the interface of the oil droplets to $\mathrm{W}_{2}$, but no evidence is found that complete $\mathrm{W}_{1}$-droplets break the oil droplet's interface. A rearrangement of the remaining $\mathrm{W}_{1}$ droplets toward the droplet's boundary occurs on a longer time scale as the system aims for concentration equilibrium. This process can be observed until the $\mathrm{W}_{1}$-droplets disappeared completely.
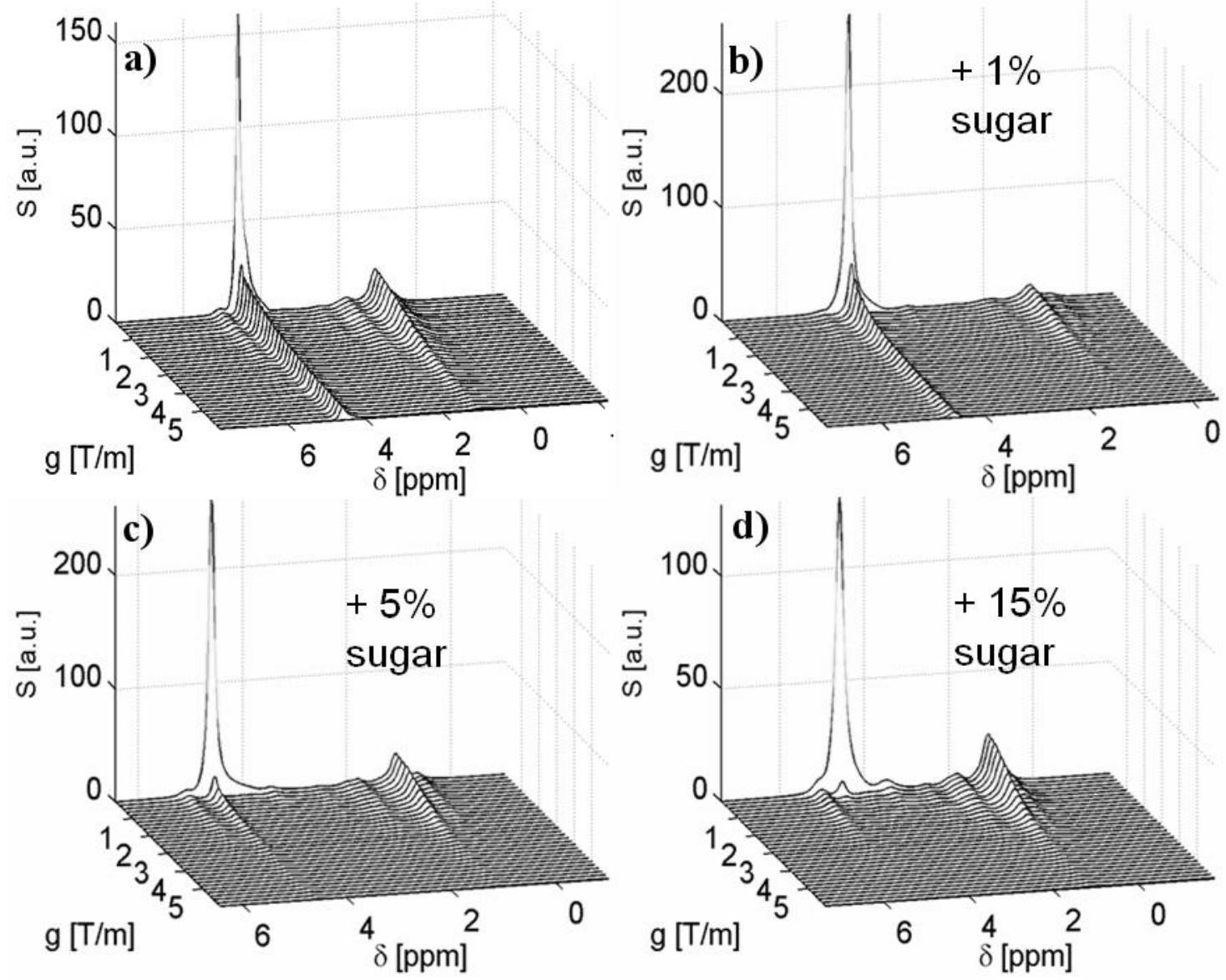

Figure 4 NMR spectra of a) a WOW double emulsion wit Xanthan in $W_{2}, b$ ) the same emulsion with additional $W_{2}$ containing $1 \%$ sugar, c) $5 \%$ sugar, d) $15 \%$ sugar. 

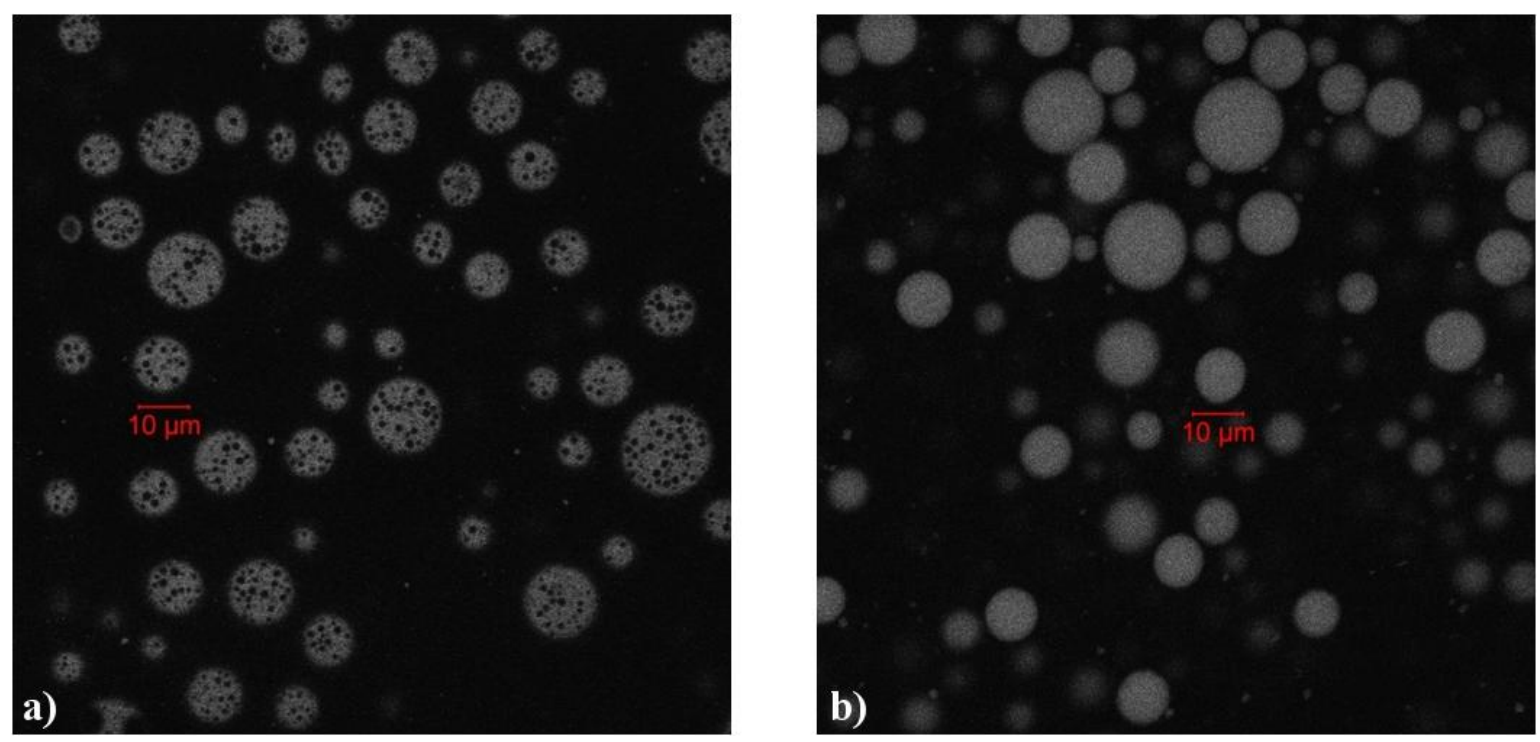

Figure 5 CLSM pictures of a) a $W_{1} / O / W_{2}$ double emulsion with Xanthan in $W_{2}$ before adding additional $W_{2}$ with $15 \%$ sugar. b) a $W_{1} / O / W_{2}$ double emulsion with Xanthan in $W_{2}$ after adding additional $W_{2}$ with $15 \%$ sugar.

2.2.4 Two Compartment Model. With the knowledge from these experiments, it is possible to separate the NMR signals of $\mathrm{W}_{1}$ and $\mathrm{W}_{2}$ in a $\mathrm{W}_{1} / \mathrm{O} / \mathrm{W}_{2}$ emulsion with Xanthan in $\mathrm{W}_{2}$. A two compartment model is applicable. The two water phases can be regarded as independent and completely separate on the experimental time scale on which the exchange is negligible. The total water signal is observed near $g=0 \mathrm{~T} / \mathrm{m}$. At higher gradient amplitudes, $\mathrm{W}_{2}$ is negligible or even not measurable. To eliminate the signal of outer water, the measured points over $q^{2}$ can be cut at the second gradient step. Discarding the signal near $g=0 \mathrm{~T} / \mathrm{m}$, the signal of $\mathrm{W}_{1}$ can now be processed with the MC-model. Therefore, the inner water phase of double emulsions of the type $\mathrm{W}_{1} / \mathrm{O} / \mathrm{W}_{2}$ can be characterised, and its DSD is found to be consistent with other measuring techniques. 


\section{CONCLUSIONS}

PFG-NMR is a suitable method to characterise $\mathrm{W}_{1} / \mathrm{O} / \mathrm{W}_{2}$ double emulsions with respect to DSD and molecular exchange phenomena. Due to the molecular exchange between both water phases, a characterisation with the common models as the MC-model is not generally possible. With Xanthan in $\mathrm{W}_{2}$ of $\mathrm{W}_{1} / \mathrm{O} / \mathrm{W}_{2}$ double emulsions, a two compartment model is applicable. The water phases can be regarded as independent. Thereby, the separated signal of the inner water phase can be evaluated by the MC-model applicable and known from investigations of single emulsions. In case of other $\mathrm{W}_{1} / \mathrm{O} / \mathrm{W}_{2}$ recipes, the exchange model can successfully be applied, revealing the DSD of the inner emulsions.

\section{Acknowledgements}

The 'Shared Research Group 10-2' received financial support by the 'Concept for the future' of Karlsruhe Institute of Technology within the framework of the German Excellence Initiative. Additional support was given the DFG, which are also highly appreciated. We would like to thank K. Sachsenheimer for help during the NMR experiments and Lydia Schütz for emulsion preparation. Special thanks to Azad Emin for help with the CLSM experiments.

\section{References}

1 P. T. Callaghan, Principles of nuclear magnetic resonance microscopy, Clarendon Press, Oxford, 1991.

2 M. L. Johns and K. G. Hollingsworth, Progress in NMR Spectroscopy, 2007, 50, 51.

3 J. S. Murday, R. M. Cotts, J. Chem. Phys., 1968, 48, 4938.

4 C. H. Neuman, J. Chem. Phys., 1974, 60, 4508.

5 J. Pfeuffer, U. Flögel, D. Leibfritz, NMR Biomed., 1998, 11, 11.

6 J. Pfeuffer, U. Flögel, D. Leibfritz, NMR Biomed., 1998, 11, 19.

7 W. S. Price, A.V. Barzykin, K. Hayamizu, M. Tachiya, Biophys. J., 1998, 74,2259.

8 B. Balinov, O. Weman, J.C. Ravey, J. Phys. Chem., 1994, 98, 393.

9 J. P. Hindmarsh, J. Su, J. Flanagan, H. Singh, Langmuir 2005, 21, 9076.

10 F. Wolf, L. Hecht, H. P. Schuchmann, E. H. Hardy, G. Guthausen, Eur. J. Lipid Sci. Technol., 2009, 111, 730.

11 X. Guan, K. Hailu, G. Guthausen, F. Wolf, R. Bernewitz, H. P. Schuchmann, Eur. J. Lipid Sci. Technol., 2010, 112, 828.

12 G. Muschiolik, I. Scherze, P. Preissler, J. Weiss et al., Multiple emulsions - preparation and stability, 13th World Congress of Food Science \& Technology 'Food is Life', 2006, $17-21$. 